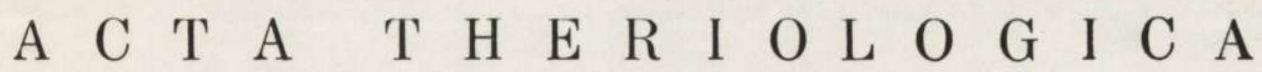 VOL. XII, 8: 93-103.

\section{Die Olive (Nucleus olivaris) des Wildschweines}

\author{
[Mit 2 Tafeln
}

Die Untersuchungen wurden auf Paraffinquerschnitten (Stärke von $15 \mu$ ) der Nachhirne von Wildschweinen durchgeführt. Die Querschnitte wurden mit Methylenblau nach der vereinfachten Methode von Nissel gefärbt. In vorliegender Arbeit sind Topographie-, Gestaltung- und Zellbau der Olive des Wildschweines beschrieben worden. Sie besteht ähnlich wie bei anderen Sảugetieren aus drei Teilen: der medialen Nebenolive, der dorsalen Nebenolive und der Hauptolive. Die mediale Nebenolive macht $93,4 \%$ des ganzen Kernkomplexes aus. Sie bildet den kaudalen Pol des Kernes, aber sie reicht jedoch nicht bis zum vorderen Pol. Die dorsale Nebenolive beträgt $91,4 \%$ des ganzen Kernes, aber sie hat keinen Anteil in der Gestaltung des kaudalen Poles des Kernes, dagegen bildet sie gemeinsam mit der Hauptolive den vorderen Pol. Die Hauptolive macht $46,5 \%$ der Kernlänge aus und bildet seinen vorderen Teil. Die erhaltenen Untersuchungsergebnisse wie auch die Vergleichung des Baues der Olive des Wildschweines mit solch einem Kern bei Haustieren scheint davon zu zeugen, dass das Wildschwein eine bedeutend primitiver ausgebildete Olive besitzt als das Schwein, und dass ihre Rolle als Koordinationszentrum im Verlauf der extrapyramidalen Bahnen bei diesem Tier bedeutend Kleiner ist als beim Schwein.

\section{EINFÜHRUNG}

Die Olive der Säugetiere besteht aus drei Teilen: der medialen Nebenolive, der dorsalen Nebenolive und der Hauptolive. Alle diese Teile der Olive bilden ein Zentrum, das mit der Erhaltung des Gleichgewichtes und der Bewegungsmöglichkeit des Säugetierkörpers verbunden ist. Die Rolle der einzelnen Teile der Olive ist dagegen bis jetzt noch nicht genau aufgeklärt worden und die über dieses Thema herrschenden Ansichten leiten ihren Ursprung hauptsächlich aus der Analyse des Ausbildungsgrades des besprochenen Kernes bei den einzelnen Säugetierarten ab. Die in vorliegender Arbeit begonnenen Untersuchungen über den Gestaltungsgrad der einzelnen Teile der Olive bei in freier Wildbahn lebenden Tieren, die mit den bisher untersuchten domestizierten Tieren nahe verwandt sind, könnten möglicher Weise das Ziehen von weiter gehenden Tätigkeitsfolgerungen erlauben. Diese 
können nämlich das Vergleichen von morphologischen Unterschieden der Olive in Betreff der Bewegungsmöglichkeit der Extremitäten und der Wirbelsäule mit nahe verwandten Tierarten aber bei einer anderen Lebensweise ermöglichen.

Vorliegende Arbeit stellt die erste Untersuchungsetappe dar, die als Einführung zum Kennenlernen der Tätigkeit der einzelnen Teile der Olive dienen soll, denn genauere Resultate werden erst wahrscheinlich experimentale Untersuchungen erbringen können, die jedoch anfänglich eine genaue und allseitige Erkenntnis der morphologischen Struktur der zu untersuchenden Zentren benötigen. Bis jetzt wurden experimentale Untersuchungen über die Olive in sehr geringen Ausmassen durchgefüht (Brouver \& Coenen 1919; Yoschimura, 1910; Brodal, 1940), aber die Resultate, die hierbei erreicht worden sind, sind noch nicht vollständig zufriedenstellend. Es wurde nämlich nur festgestellt, dass die Nebenoliven mit dem Paleocerebellum und die Hauptolive mit dem Neocerebellum vermittels der oliv-kleinhirnigen und der kleinhirnig-oliven Bahnen, aber mit dem Rückenmark durch rückenmarkig-olive und oliv-rückenmarkige Bahnen verbunden sind. Dies bestätigte die Untersuchungsergebnisse über die Ontogenese der Olive ( $\mathrm{R}$ u ciński \& Welento, 1958; Ziolo, 1966), deren Ansicht nach die Nebenoliven entogenetisch und phylogenetisch (K a p pers, 1960) ältere Gebilde sind als die Hauptolive.

Als Untersuchungsmaterial dienten Nachhirne von Wildschweinen, Sus scrofa L in na eus, 1788, die vom Woiwodschaftsrat für Jagdwesen in Lublin zur Verfügung gestellt wurden. Die Untersuchungen selbst wurden auf Querschnittserien von $15 \mu$ Stärke durchgeführt, wobei jeder dritte Querschnitt untersucht wurde. Die Präparate wurden mit Methylenblau von Löffler nach der vereinfachten Methode von Nissel gefärbt.

\section{EIGENE UNTERSUCHUNGEN}

Der Kernkomplex der Olive beim Wildschwein ist eine Anhäufung von Nervenzellen - gelegen im ventro-medialen Teil der Medulla oblongata im Abschnitt von der Querfläche, die in Entfernung zirka eines mm nach vorn vom kaudalen Pol des Kernes des N. hypoglossus durchgeführt ist, bis zur Fläche, die in Entfernung zirka 0,6 $\mathrm{mm}$ nach vorn vom kaudalen Pol des Kernes des $N$. facialis verläuft. Beim Wildschwein ist etwas mehr als die Hälfte des Nucleus olivaris anterior und etwas weniger als die Hälfte posterior vom Calamus scriptorius gelegen. Die Olivenlänge betrug bei den untersuchten Tieren durchschnittlich $11,5 \mathrm{~mm}$. In der Olive des Wildschweines kann man ähnlich wie bei anderen Säugern 3 Teile unterscheiden: die mediale Nebenolive $(N$. olivaris accessorius medialis), die dorsale Nebenolive ( $N$. olivaris accessorius dorsalis) und die Hauptolive ( $N$. olivaris principalis).

1. Nucleus olivaris accessorius mediaiis (om, ldm, lvm, Abb. 1-10 und 13)

Die mediale Nebenolive nimmt im Olivenkomplex eine ventro-mediale Lage ein. Sie reicht kaudal weiter als die dorsale Nebenolive und die 
Hauptolive, aber dies verursacht, dass der kaudale Abschnitt des Nucleus olivaris $(8,1 \%$ seiner Länge) nur durch die mediale Nebenolive gebildet wird und dass der kaudale Pol des Nucleus olivaris accessorius medialis gleichzeitig der kaudale Pol der Olive ist. Anterior reicht die mediale Nebenolive nicht bis zum anterioren Pol von Nucleus olivaris - ihr anteriorer Pol ist in geringer Entfernung kaudal von der kaudalen Grenze eines zwanzigsten der vorderen Teils der Olive gelegen. Die Länge des Nucleus olivaris accessorius medialis beim Wildschwein gleicht $93,4 \%$ der Länge des Olivenkomplexes.

Die mediale Nebenolive bilden zwei Zellstränge: die ventrale Lamelle und die dorsale Lamelle. Die ventrale Lamelle liegt schräg von der dorso-medialen Seite zur ventro-lateralen Seite. Sie ist ein flacher langer Zellstrang, der von dem anterioren bis zum kaudalen Pol der medialen Nebenolive reicht. Auf den Querschnitten ist die ventrale Lamelle eine schmale verlängerte Zellgruppe, die sich verkleinert und die Gestalt eines Ovales in der Nähe des anterioren und kaudalen Poles annimmt. Im anterioren Abschnitt ist die ventrale Lamelle eine deutlich dickere und mehr zusammengedrängte Zellanhäufung als im kaudalen Abschnitt.

Die ventrale Lamelle der medialen Nebenolive begleiten zwei Zellstränge. Sie sind auf den Querschnitten in Gestalt von abgerundeten Zellgruppen sichtbar, die auf der inferioren Seite der ventralen Lamelle der medialen Nebenolive gelegen sind. Der eine der beschriebenen Zellstränge begleitet den kaudalen - und der zweite den mittleren Abschnitt der ventralen Lamelle, wobei der kaudale Strang medialer gelegen ist als der anteriore.

Die dorsale Lamelle der medialen Nebenolive ist im Vergleich mit der ventralen Lamelle bedeutend schlechter ausgebildet. Sie besteht aus einem verhältnismässig kurzen Zellstrang. Sie reicht von der Gegend der anterioren Grenze - eines Zehntels der kaudalen Teils, bis zur Gegend der kaudalen Grenze - zwei Fünftels - der anterioren Teils der medialcn Nebenolive. Die dorsale Lamelle ist senkrecht, parallel zur Naht in ihrer Nähe gestellt. Die Lamina dorsalis liegt superior von dem dorso-medialen Rand der ventralen Lamelle, mit der sie vermittels ihrer ventralen Fläche unter einem stumpfen Winkel kontaktiert. Von der medialen Seite wird die dorsale Lamelle durch Längsfurchen gerieft, die auf den Querschnitten in Gestalt von tiefen Kerben sichtbar sind. An vielen Stellen sind diese Kerben so tief, dass sie die dorsale Lamelle auf abgesonderte, senkrecht aufeinander gelegene Zellgruppen aufteilen.

Die mediaie Nebenolive ist aus ziemlich dicht aber unregulär gelegenen, sich dunkel färbenden vielpoligen Zellen gebildet und zwar dreieckigen und abgerundeten mit Ausmassen von 17 bis $29 \mu$. In der medialen Nebenolive findet man gleichfalls einzelne spindelförmige Zellen. Diє 
Zellen der medialen Nebenolive enthalten dicke Körner einer tigroidalen Substanz. Die Zellkerne sind gross, abgerundet und besitzen ein zentral gelegenes Kernkörperchen. In der dorsalen Lamelle der medialen Nebenolive sind die Zellen loser angeordnet als in der ventralen Lamelle. In den Zellsträngen, die die ventrale Lamelle der medialen Nebenolive begleiten, sind die Zellen grösser als im Nucleus olivaris accessorius medialis und ausserdem treten dort zahlreiche spindelförmige Zellen auf.

2. Nucleus olivaris accessorius dorsalis (od, pad, ppd, Abb. 2-12 und 14)

Die dorsale Nebenolive ist dorsal und lateral von den übrigen Teilen des Nucleus olivaris gelegen. Sie zieht sich von der in geringer Entfernung kaudal von der anterioren Grenze eines Zehntels von Nucleus olivaris durchgeführten Querfläche, bis zum anterioren Pol der Olive. Die Länge des Nucleus olivaris accessorius dorsalis gleicht beim Wildschwein $91,4 \%$ der Länge des ganzen Kernkomplexes der Olive.

In der dorsalen Nebenolive kann man zwei Teile unterscheiden: Pars posterior und Pars anterior. Der kaudale Pol von Pars posterior liegt in der Nähe des superioren Randes der lateralen Fläche von der dorsalen Lamelle der medialen Nebenolive. Von dieser Stelle ab richtet sich der Zellstrang, den kaudalen Teil der dorsalen Nebenolive bildend, anterior wie auch ventral und lateral. Mit seinem anterioren Ende verbindet er sich sehr eng mit dem kaudalen Pol des anterioren Teiles der dorsalen Nebenolive. Die Verschmelzung der beiden Teile der dorsalen Nebenolive liegt dorsal und lateral von der ventralen Lamelle der medialen $\mathrm{Ne}$ benolive kaudal von der halben Länge des Nucleus olivaris. Der vordere Teil der dorsalen Nebenolive ist ein flacher Zellstrang, der sich von der Verbindungsstelle mit dem kaudalen Teil anterior sowie dorsal und medial richtet. Der vordere Pol von Pars anterior liegt vom vorderen Pol der Hauptolive dorsal und medial.

Auf den Querschnittbildern ist Pars anterior oval und weiter in anteriorer Richtung als schmale verlängerte Zellgruppe ausgebildet, die von der dorso-medialen Seite zur ventro-lateralen Seite schräg gelegen ist und die sich stufenweise in ventro-lateralen Richtung verschiebt. Der vordere Abschnitt von Pars posterior ist eng mit dem kaudalen Pol von Pars anterior verbunden und auf Querschnittbildern sieht er wie eine abgerundete Zellgruppe aus, die dorsal und lateral von der ventralen Lamelle der medialen Nebenolive gelegen ist.

Der anteriore Teil der dorsalen Nebenolive ist auf den Querschnittbildern eine schmale verlängerte Zellgruppe, die schräg von der dorso-medialen Seite zur ventro-lateralen Seite parallel zum Brachium superior der Hauptolive auf seiner dorso-lateralen Seite liegt. Im Mittel- 
abschnitt biegt sich der dorso-mediale Rand von Pars anterior medial und ventral in Richtung von Brachium superior der Hauptolive ein, mit dem er sich nach einer kurzen Strecke vereinigt. In diesem Abschnitt befindet sich zwischen der dorsalen Nebenolive und Brachium superior der Hauptolive ein schmaler Sinus ohne jeglichen Zellen, der von der dorso-medialen Seite geschlossen, aber von der ventro-lateralen Seite geöffnet ist. In der Nähe des anterioren und kaudalen Poles nimmt der vordere Teil der dorsalen Olive auf den Querschnittbildern die Gestalt einer abgerundeten Zellgruppe an. Kaudal verbindet sie sich mit dem kaudalen Teil der dorsalen Nebenolive, aber anterior dagegen verkleinert sie sich stufenweise und unterliegt auf der ventro-medialen Seite des vorderen Poles der Hauptolive dem Schwund; mit ihm gemeinsam bildet sie aber den anterioren Pol von Nucleus olivaris.

Die dorsale Nebenolive bilden lose und unregulär angeordnete dreiekkige, vielpolige und abgerundete Zellen mit Ausmassen von $17-30 \mu$. Diese Zellen enthalten zahlreiche dicke Körner von tigroidaler Substanz und grosse kugelige Kerne mit einem zentral placierten Kernkörperchen. In der dorsalen Nebenolive treten ebenfalls vereinzelte spindelförmige Zellen auf, die in Pars posterior verhältnismässig zahlreicher sind als in Pars anterior. Der Färbungsintensität nach unterscheiden sich die Zellen der dorsalen Nebenolive nicht von denjenigen der medialen Nebenolive, aber sie sind jedoch loser angeordnet als in der Letzteren.

3. Nucleus olivaris principalis

(op, bip, bsp, Abb. 7-12 und 15)

Die Hauptolive liegt in der anterioren Hälfte des Olivenkomplexes zwischen dem vorderen Teil der dorsalen Nebenolive und der ventralen Lamelle der medialen Nebenolive. Der kaudale Pol der Hauptolive liegt in geringer Entfernung kaudal von der kaudalen Grenze neun Zwölftels der vorderen Teils von Nucleus olivaris. Der anteriore Pol von Nucleus olivaris principalis bildet gemeinsam mit dem vorderen Pol von Nucleus olivaris accessorius dorsalis den vorderen Pol des Olivenkomplexes. Die Länge der Hauptolive beim Wildschwein gleicht 46,5\% der Länge von Nucleus olivaris.

Die Hauptolive wird von zwei parallelen Zellsträngen gebildet: dem Oberarm (Brachium superior) und dem Unterarm (Brachium inferior). Die Arme der Hauptolive sind schräg von der dorso-medialen Seite zur ventro-lateralen Seite, parallel zum vorderen Teil der dorsalen Nebenolive und der ventralen Lamelle der medialen Nebenolive gelegen. Der Oberarm der Hauptolive liegt ventral und medial von dem anterioren Teil der dorsalen Nebenolive, aber der Unterarm ist dorsal und lateral von der ventralen Lamelle der medialen Nebenolive gelegen. Die ventro- 
-lateralen Ränder beider Arme der Hauptolive sind mit einander verbunden und zwischen ihnen befindet sich ein schmaler zellfreier Sinus, der von der dorso-medialen Seite geöffnet, aber von der ventro-lateralen Seite geschlossen ist. Im Mittelabschnitt der Hauptolive vereinigt sich ihr Oberarm auf einer kurzen Strecke mit dem in seiner Richtung eingebogenen dorso-medialen Rand des vorderen Teiles der dorsalen Nebenolive. Kaudal nehmen beide Arme der Hauptolive ihren Anfang auf derselben Höhe, aber anterior unterliegt der Oberarm etwas früher dem Schwund als der Unterarm, der seinerseits den anterioren Pol der Hauptolive selbständig ausbildet. Der vordere Pol des Unterarmes liegt ventral und lateral vom vorderen Pol des anterioren Teiles der dorsalen Nebenolive und beide letztens erwähnten Zellanhäufungen bilden den anterioren Pol von Nucleus olivaris.

Auf den Querschnittbildern erinnert die Hauptolive ihrer Gestalt nach an einen schräg angeordneten Buchstaben »U«, der mit der geschlossenen Seite ventro-lateral, aber mit der geöffneten dorsal und medial gerichtet ist. Auf einer kurzen Strecke im Mittelabschnitt der Hauptolive unterliegt diese Gestalt einer gewissen Veränderung, denn der ventro-laterale Rand der Hauptolive biegt sich ventral und medial in der Richtung der ventralen Lamelle der medialen Nebenolive ein. Der anteriore kurze Abschnitt der Hauptolive, der nur durch den ventralen Arm gebildet wird, ist auf den Querschnittbildern eine schmale verlängerte Zellgruppe, die in der Richtung des vorderen Poles die Gestalt eines Ovales annimmt, aber die sich schnell verringert und auf der Höhe des anterioren Poles des vorderen Teilen der dorsalen Nebenolive auf seiner ventro-lateralen Seite dem Schwund unterliegt. Die Hauptolive wird von ziemlich dicht angeordneten dreieckigen, vielpoligen, abgerundeten und spindelförmigen Zellen mit Ausmassen von 21 bis $34 \mu$ gebildet. Die Zellkerne sind gross, kugelig mit einem zentral placierten Kernkörperchen. In der Hauptolive sind, ähnlich wie in der medialen Nebenolive, die Zellen deutlich dichter angeordnet als in der dorsalen Nebenolive. Die Färbungsintensität der Zellen der Hauptolive unterscheidet sich jedoch nicht weder von denjenigen der dorsalen Nebenolive noch von der medialen Nebenolive.

\section{BESPRECHUNG}

Den Gestaltungsgrad der Olive von in freier Wildbahn lebenden Tieren im Vergleich zu eben diesem Kern bei domestizierten Tieren hat Vermeulen (1924) untersucht. Seiner Ansicht nach gilt als charakteristisches Merkmal für alle Kerne der Medulla oblongata bei in freier Wildbahn lebenden Tieren die Tatsache, dass sie mehr kaudal im Ver- 
hältnis zum Calamus scriptorius gelegen sind als bei domestizierten Tieren, und dass mit dem Fortschreiten der Stufe der Domestizierung der Tiere - diese Kerne sich nach vorn verschieben. Die in unserem Katheder durchgeführten Untersuchungen über die Lage der Olive bei Tieren haben die Ansicht von Vermeulen (1924) nur teilweise bestätigt.

Der vor Calamus scriptorius gelegene Prozentsatz:

$\begin{array}{ll}\text { Pferd (M i l a t, 1965) } & 75 \% \\ \text { Schaf (S z t y n, 1966) } & 66 \% \\ \text { Schwein (eigene Beobachtungen auf dem } & \\ \text { Material von L e w a n dowski, 1952) } & 52 \% \\ \text { Wildschwein } & 52 \% \\ \text { Ziege (S z e y n \& W e l e n t o, in litt.) } & 40 \% \\ \text { Nutria (S zte y n, 1965) } & 25 \%\end{array}$

Aus obigen Angaben scheint hervorzugehen, dass die Lage der Olive weniger vom Grade der Domestizierung abhängig ist, als dies auf der Grundlage der Arbeit von Verme u le n (1924) zu sein schien. Freilich liegt die Olive bei Domestizierten Tieren mehr anterior als bei der Nutria, aber die Lage dieses Kernes beim Schwein und Wildschwein ist identisch.

Die Vergleichung des Baues der einzelnen Oliventeile bei beiden letzten Tieren hat erwiesen, dass sie gänzlich verschieden ausgebildet sind.

Der Längenprozentsatz der einzelnen Teile der Olive beim Schwein und Wildschwein ist:

$\begin{array}{lccc} & \text { Hauptolive } & \text { Mediale Nebenolive } & \text { Dorsale Nebenolive } \\ \text { Schwein (Lewandowski, 1952) } & 50,0 & 85,0 & 75,0 \\ \text { Wildschwein } & 46,5 & 94,4 & 91,4\end{array}$

Die schwächere Entwicklung der Hauptolive beim Wildschwein (im Vergleich zum Schwein) scheint beim Wildschwein eine schwächere Entwicklung seiner Verbindungen mit dem Neocerebellum zu suggerieren mit dieses Teil des Kleinhirnes dieser Kern sich vereinigt (B r oda l, 1940; Brouver \& Coenen, 1919; Kappers, 1960). Die Untersuchungen von Larsell \& Berthelsdorf (1941) über die Verknüpfung des Kleinhirns mit der Muskulatur des Rumpfes und der Extremitäten haben erwiesen, dass Tiere, die sich durch eine bessere Koordination der Arbeit der Gliedmassenmuskeln mit den Rumpfmuskeln kennzeichnen, ein besser ausgebildetes Neocerebellum besitzen. $\mathrm{Da}$ anderseits vermutet wird, dass die Hauptolive mit den Gliedmassen in Verbindung steht (B r unner, 1917; K a ppers, 1960; Szteyn, 1965) scheint es, dass die schwache Entwicklung der Hauptolive beim Wildschwein von einer schwächeren Koordination der Muskelarbeit des Rumpfes mit derjenigen der Gliedmassen bei diesem Tier zeugen kann, 
aber auch davon, dass die letztgenannte Tätigkeit die Hauptfunktion der Hauptolive sein kann.

Einen besseren Entwicklungsgrad weist dagegen beim Wildschwein (im Vergleich zum Schwein) die mediale - und dorsale Nebenolive auf.

Die mediale Nebenolive dagegen ist beim Wildschwein bedeutend besser ausgebildet als bei Haustieren (K old a, 1928; L e wa n d owski, 1952; Milart, 1965; Szteyn, 1967, Szteyn \& Welento, (1967), aber etwas schwächer als bei der Katze (Y od a, 1941; T a b e r, 1961); beim Hund ( $\mathrm{O}$ oda, 1940), bei der Fledermaus ( $\mathrm{c} \mathrm{h}$ ober, 1959/60), beim Kaninchen (O ls z e w ski \& M e e s s e n, 1949) und beim Seehund (K o o y, 1917). Die Rolle der medialen Nebenolive ist eigentlich bisher noch nicht bekannt. Kappers (1960) und Lew and owski (1952) binden ihren Entwicklungsgrad mit dem Grad der Bewegungsmöglichkeit der Wirbelsäule. Brunner (1917) suggeriert ihre Verbindung mit der vorderen Extremität, aber $\mathrm{S} z$ te y $\mathrm{n}(1965)-$ mit dem Entwicklungsgrad des Gleichgewichtssinnes. Alle diese Ansichten sind jedoch nicht genügend bewiesen worden, denn sie stützen sich nur auf die Analyse der morphologischen Olivenstruktur im Vergleich zur Lebensweise und der Bewegungsart der untersuchten Tiere. Es scheint jedoch, dass die entgültige Klärung der Rolle dieses Kernes erst die Ergebnisse der experimentalen Untersuchungen erbringen werden.

Auf Grund vorliegender Untersuchungen und derjenigen anderer Autoren über die Olive (B r o d a l, 1940; B r o uve r \& C o e n e n, 1919) kann man jedoch feststellen, dass der Ausbildungsgrad der medialen Nebenolive beim Wildschwein eine Bedeutend bessere Verbindung dieses Kernes mit dem Paleocerebellum im Vergleich mit dem Schwein suggeriert. Die Ansicht scheint ebenfalls die Tatsache einer bedeutend besseren Ausbildung der dorsalen Nebenolive beim Wildschwein als beim Schwein zu bestätigen, weil dieser Teil der Olive sich ebenfalls mit dem Paleocerebellum vereinigt (Brouver \& Coenen, 1919; Brod a l, 1940).

Die Bedeutung der Aktivität der dorsalen Nebenolive ist sehr schwach erkannt worden und die Ansichten über dieses Thema sind demnach unzutreffend, so wie diejenigen über die Rolle der medialen Nebenolive. Brunner (1917) ist der Meinung, dass die Entwicklung der dorsalen Nebenolive mit dem Bewegungsmöglichkeitsbereich der Beckenextremität verbunden ist, aber $\mathrm{Kappers}(1960)$ dagegen schreibt $\mathrm{ihr}$ eine Funktion zu, die mit der Statik und Lokomotion des ganzen Körpers verbunden ist. Im Zusammenhang mit der bedeutend besseren Entwicklung der dorsalen Nebenolive beim Wildschwein (91,4\% Länge des ganzen Olivenkomplexes) als beim Schwein $(75 \%)$ und ihrer wahrscheinlichen Verbindung mit den Beckenextremitäten, darf man vermuten, dass 
die Bewegungsleistungsfähigkeit der Beckengliedmassen des Wildschweines bedeutend grösser ist als beim Schwein

Der Bau der Olive beim Wildschwein im Vergleich zu solch einem Kern beim Schwein (L e w and ow ski, 1952) und bei anderen domestizierten Huftieren weist eine bedeutend losere Anordnung der Zellen und eine ärmere Faltung der einzelnen Lamellen auf. Dieser "primitivere Typ" des inneren Baues der Olive und der Grad der Ausbildung der einzelnen Teile der Olive scheinen von einer bedeutend kleineren Koordinationsrolle des beschriebenen Zentrums des Wildschweines im Verlauf der extrapyramidalen Bahnen des Hirnstammes und des Rückenmarkes zu deuten. Der Entwicklungsgrad der einzelnen Teile der Olive des Wildschweines scheint ebenfalls vom engeren Zusammenhang dieses Kernes mit dem Paleocerebellum anstatt mit dem Neocerebellum zu zeugen, folglich, und es könnte so sein, von einer anderen phylogenetisch niedrigeren Entwicklungsstufe des Kleinhirnes des Wildschweines als bei Haustieren.

\section{SCHRIFTTUM}

1. Brodal A., 1940: Experimentelle Untersuchungen über die Olivocerebellare Lokalisation. Ztschr. Ges. Neurol. Psychiat., 169, 1: 1-153.

2. Brouver E. \& Coenen L., 1919: Uber die Oliva inferior. J. Psych. Neurol., 25: $52-71$.

3. Brunner H., 1917: Zur Kenntnis der unteren Olive bei den Säugetieren. Arb. Neurol. Inst. Wiener Univ., 12, 1: 113-199.

4. K a p per s A., 1960: The comparative anatomy of the nervous system of vertebrates including man. Hafner Publ. Co., 1: 668-689, New York.

5. Kolda J., 1928: L'olive inférieure du boeuf. Comptes Rendeus de l'Association des Anatomistes: 1-8, Prague.

6. K o о y F., 1917: The inferior olive in vertebrates. Folia Neurobiol., 10: 205-369.

7. Larsell O. \& Berthels orf S., 1941: The ansoparamedian lobule of the cerebullum and its correlation with the limb-muscle masses. J. comp. Neurol., 75, 2: $315-340$.

8. L ew an d ow ski M., 1952: Oliwa dolna (nucleus olivaris inferior) świni. Annls Univ. M. Curie-Skłodowska, Sec. DD 6 [1951]: 294-307, Lublin.

9. Milart Z., 1965: Die Olive des Pferdes. Wiener Tierärtzl. Monatschr., 52, 4: 223-230.

10. Olszewski J. \& Meessen H., 1949: Cytoarchitektonischer Atlas des Rautenhirns des Kaninchens. S. Karger: 14-23 und 45. Basel.

11. Ruciński T. \& Welento J., 1958: Rozwój oliwy tylnej u bydła. Annls Univ. M. Curie-Skłodowska sect. DD 11 [1956]: 147-162. Lublin.

12. Schober W., 1959/60: Zur Cytoarchitektonik und Cytologie der Medulla oblongata der Mausohr-Fledermaus Myotis myotis (Borkhausen). Wissen. Ztschr. Karl Marx Univ., 9, 5: 745-771.

13. Szte y n S., 1967: Oliwa (nucleus olivaris) owcy (Ovis aries). Polskie Arch. wet., 10 , (in print). 
14. Szteyn S., 1965: Oliwa tylna (nucleus olivaris caudalis) nutrii (Myocastor coypus Molin a). Annls Univ. M. Curie-Skłodowska, Sect. C 19 [1964]: 177188. Lublin.

15. Szteyn S. \& W elento J., 1967: Oliwa (nucleus olivaris) kozy (Capra dom.). Annls Univ. M. Curie-Skłodowska, sect. DD 21, (in print).

16. T a ber E., 1961: The cytoarchitekture and cytologie of the brain stem of the cat. J. comp. Neurol., 116, 1: 27-56.

17. Vermeulen H. A., 1924: De involoed van het huisdieworden op den bouw der hersenen. Nederl. Zijdschr. Geneesk., 68, 2: 1931-1946.

18. Yo da S., 1940: Uber die Kerne der Medulla oblongata der Katze. Ztschr. mikroskop.-anat. Forsch., 48: 529-582.

19. Yoda S., 1941: Beitrag zu den Olivenkernen des Hundes. Ztschr. mikroskop.-anat. Forsch., 49: 516-524.

20. Yoschimura K., 1910: Experimentelle und vergleichende anatomische Untersuchungen über die untere Olive der Vögel. Arb. Neurol. Inst. Wiener Univ., 18, 1: 46-79.

21. Z i o ło I., 1966: Rozwój jądra oliwki świni. Folia morphol., 25, 2: 267-276.

Eingang des Ms., 30 April 1966.

Department of Animal Anatomy,

College of Agriculture,

Lublin, Akademicka 11, Poland.

Janusz WELENTO i Stanisław SZTEYN

OLIWA (NUCLEUS OLIVARIS) DZIKA

\section{Streszczenie}

Badania niniejsze zostały wykonane na podstawie parafinowych skrawków poprzecznych (grubości $15 \mu$ ) rdzeni przedłużonych dzików. Skrawki barwiono blękitem metylenowym Löfflera wg metody Nissla.

W pracy opisano topografię, ukształtowanie i budowẹ komórkową oliwy dzika. Oliwa dzika, podobnie jak u innych ssaków, utworzona jest z trzech części: przycliwy przyśrodkowej, przyoliwy grzbietowej i oliwy głównej. Przyoliwa przyśrodkowa zajmuje w kompleksie jądra oliwy położenie brzuszno-przyśrodkowe i sięga dalej ku tyłowi niż oliwa główna i przyoliwa grzebietowa. Długość przyoliwy przyśrodkowej dzika równa się 93,4\% długości calego jądra. Przyoliwa grzbietowa położona jest grzbietowo i bocznie od oliwy głównej i przyoliwy przyśrodkowej. Przyoliwa grzbietowa w stosunku do przyoliwy przyśrodkej jest przesunięta do przodu i sięga do przedniego bieguna oliwy. Długość przyoliwy grzebietowej dzika stanowi 91,4\% długości nucleus olivaris. Oliwa główna leży między przyoliwą grzebietową i przyoliwą przyśrodkową w przedniej połowie nucleus olivaris. Oliwa główna, podobnie jak przyoliwa grzebietowa, sięga dalej w kierunku przednim niż przyoliwa przyśrodkowa, osiągając przedni biegun nucleus olivaris. Długość oliwy głównej dzika równa się $46,5 \%$ długości całego kompleksu. 
Oliwa dzika utworzona jest z komórek wielobiegunowych, trójkątnych i zaokrąglonych, które w oliwie głównej i przyoliwie przyśrodkowej ułożone są gęściej niz w przyoliwie grzebietowej. W intensywności zabarwienia komórki poszczególnych części nucleus olivaris nie wykazują różnic.

Uzyskane wyniki badań w porównaniu do budowy oliwy świni oraz innych domowych zwierząt kopytnych wykazują, iż oliwa dzika posiada bardziej ,prymitywny" typ budowy wewnętrznej. Posiada ona bowiem znacznie luźniejsze ułożenie komórek i uboższe pofałdowanie blaszek. Potwierdza ten wniosek także fakt, iż stopień wyksztalcenia poszczególnych części oliwy jest u dzika odmienny niż u świni i wydaje się świadczyć o znacznie mniejszej roli koordynacyjnej opisywanego ośrodka dzika w przebiegu szlaków pozapiramidowych pnia mózgu i rdzenia kręgowego. Stopień rozwoju poszczególnych części oliwy dzika może świadczyć także o ściślejszym związku tego jądra z paleocerebellum niż z neocerebellum, a więc może także o odmiennym niż u zwierząt domowych, niższym filogenetycznie stopniu rozwoju móżdżku dzika. 


\section{TAFELBESCHREIBUNG}

\section{Tafeln VI-VII.}

Abb. 1-12. Querschnitte der Olive des Wildschweines aus der Gegend des kaudalen Poles (Abb. 1) bis zu derjenigen des anterioren Poles (Abb. 12). Vergr. $14 \times$.

bip - Brachium inferior Nucleus olivaris principalis, bsp - Brachium superior Nucleus olivaris principalis, kt - Zellstränge die die Lamina ventralis Nucleus olivaris accessorius medialis begleiten.

1 - laterale Seite, Idm - Lamina dorsalis Nucleus olivaris accessorius medialis, lvm - Lamina ventralis Nucleus olivaris accessorius medialis,

m - mediale Seite, od - Nucleus olivaris accessorius dorsalis, om - Nucleus olivaris accessorius medialis, op - Nucleus olivaris principalis,

pad - Pars anterior Nucleus olivaris accessorius dorsalis, ppd - Pars posterior Nucleus olivaris accessorius dorsalis, $\mathrm{r}$ - Raphe, sd - Sinus nucleus olivaris accessorius dorsalis, sp - Sinus nucleus olivaris principalis.

Abb. 13. Vergr. $300 \times$. Zellen der medialen Nebenolive.

Abb. 14. Vergr. $300 \times$. Zellen der dorsalen Nebenolive.

Abb. 15. Vergr. $300 \times$. Zellen der Hauptolive. 


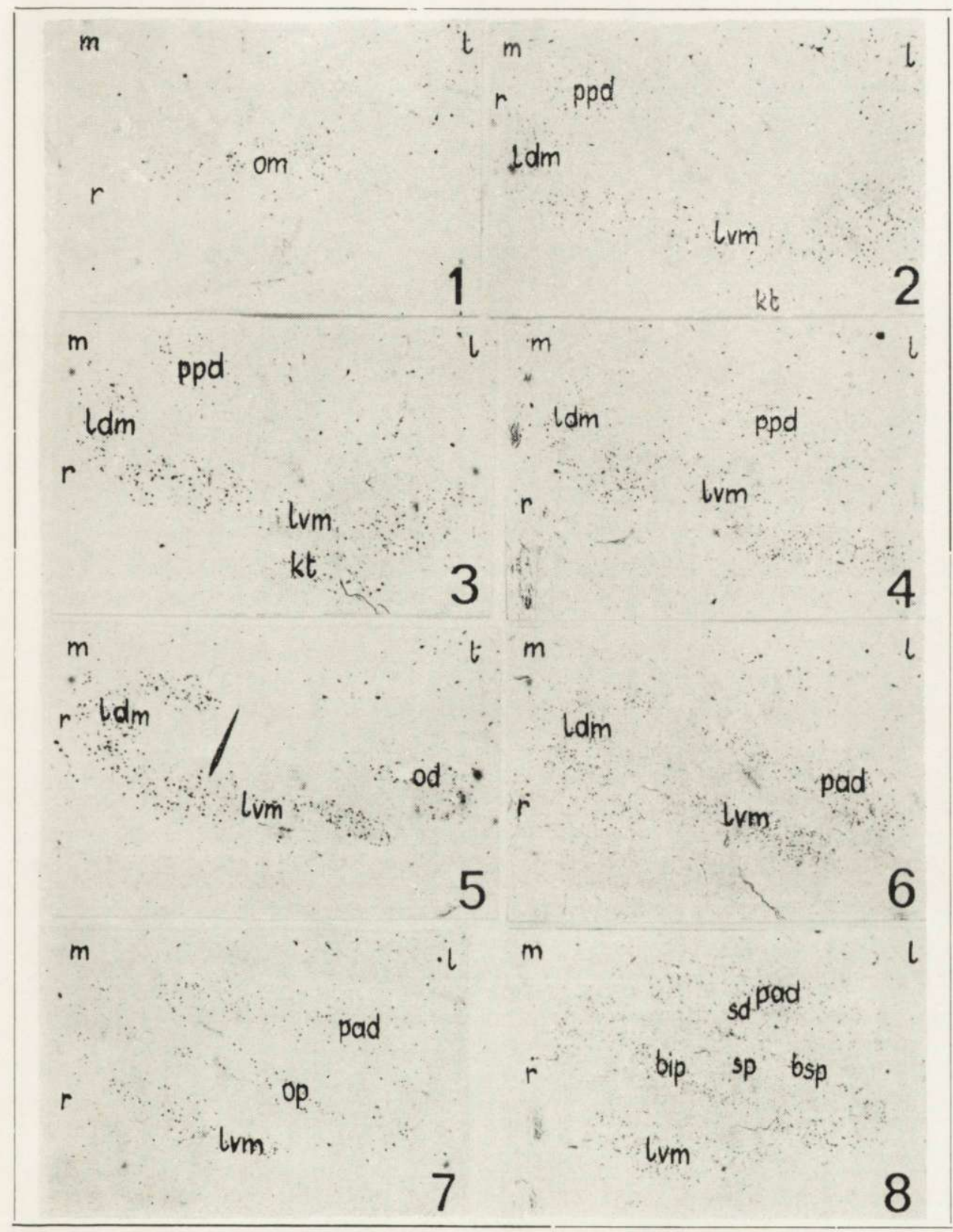

J. Welento \& S. Szteyn

auctores phot. 
ACTA THERIOLOGICA, Vol. XII, 8.

Tafel VII.

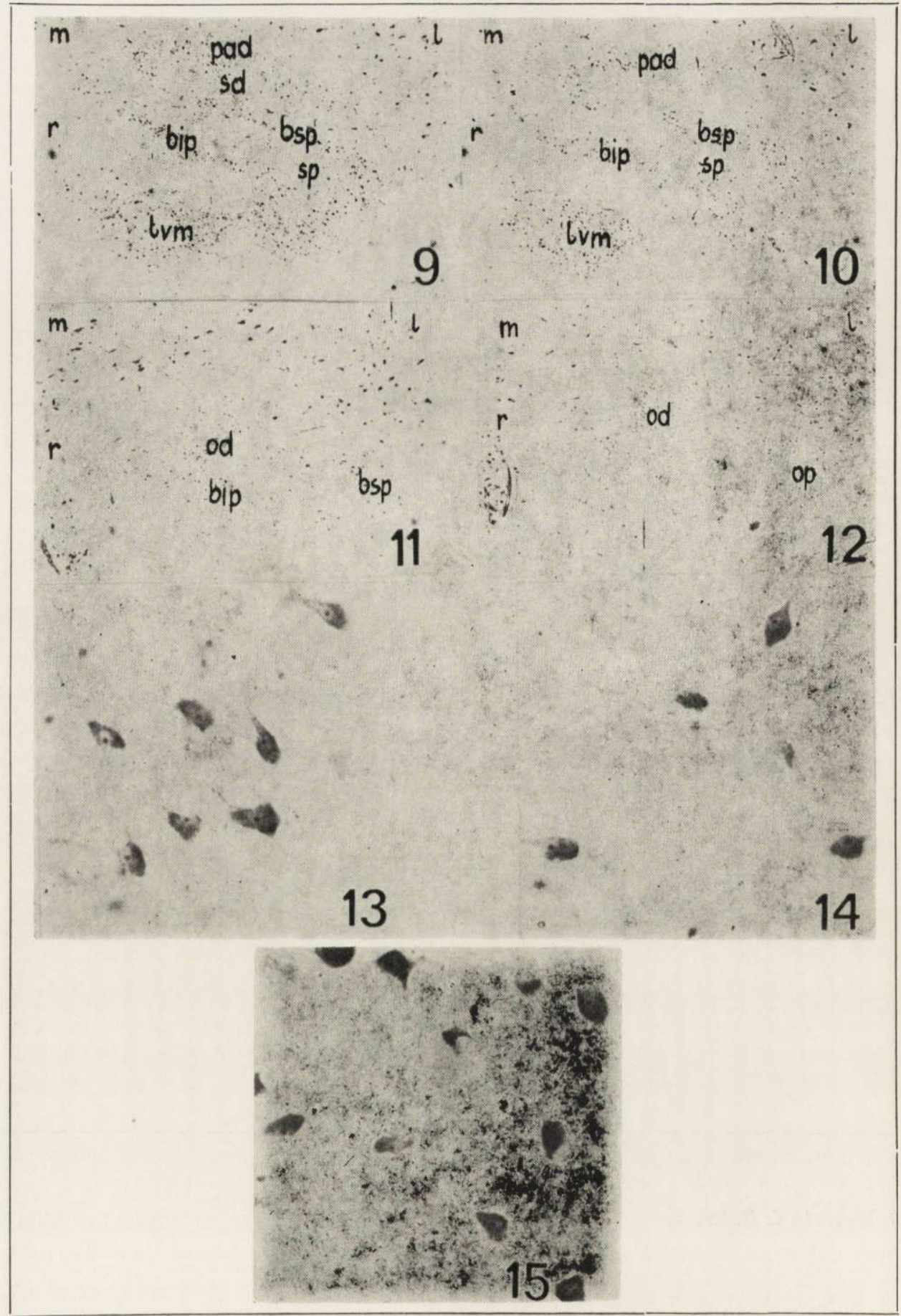

J. Welento \& S. Szteyn

auctores phot. 